\title{
A Phasor-Data-Based State Estimator Incorporating Phase Bias Correction
}

\author{
Luigi Vanfretti, Member, IEEE, Joe H. Chow, Fellow, IEEE, Sanjoy Sarawgi, Member, IEEE, and \\ Behruz (Bruce) Fardanesh, Senior Member, IEEE
}

\begin{abstract}
With amplitude and phase information, time-synchronized measured phasor data of bus voltages and line currents can be used to calculate, without iterations, the voltage phasor on neighboring buses. In some phasor measurement units (PMUs), it has been observed that the voltage and current phasors exhibit phase biases, which can corrupt the conventional state estimator solution if it is augmented with such biased phasor data. This paper presents a new approach for synchronized phasor measurement-based state estimation, which can perform phasor angle bias correction given measurement redundancy. In this approach, polar coordinates are used as the state variables, because the magnitude and phase are largely independent measurements. The state estimation is formulated as an iterative least-squares problem, and its application to portions of the AEP high-voltage transmission system is illustrated.
\end{abstract}

Index Terms-Bad data correction, observability, phasor measurement redundancy, PMU data accuracy, state estimation, synchronized phasor measurements.

\section{INTRODUCTION}

$\mathbf{S}$ TATE estimation [1]-[4] provides static estimates of the system states using bus voltage magnitude and line active and reactive power flow measurements. Currently state estimators (SEs) use the Intercontrol Center Communications Protocol (ICCP) to gather asynchronously fed data with an arrival rate of 1 sample per 4-10 s. Even though there have been great efforts to improve the detection of bad data and topology errors [2], [3], [5], there is still a need to provide better bad data detection. With the advancement of synchrophasor technology, there has been interest to use phasor measurement unit (PMU) measurements to enhance the accuracy provided by SEs [6]. Inclusion of phasor measurements within static SEs has been reported by several utilities [7]-[10]. The penetration of PMU measurements compared to conventional measurements is, however, still too limited to have a noticeable impact on the SEs solution [11].

Manuscript received July 10, 2009; revised January 12, 2010. First published April 26, 2010; current version published January 21, 2011. This work was supported in part by the RPI Power System Research Consortium Industry Members: AEP, FirstEnergy, NE-ISO, NYISO, and PJM, and in part by theNSF under grant ECS-0622119. Paper no. TPWRS-00532-2009.

L. Vanfretti and J. H. Chow are with the Department of Electrical, Computer, and Systems Engineering, Rensselaer Polytechnic Institute, Troy, NY 12180 USA (e-mail: vanfretti@alum.rpi.edu; chowj@rpi.edu).

S. Sarawgi is with American Electric Power, Gahanna, OH 43230 USA (e-mail: sksarawgi@aep.com).

B. Fardanesh is with the New York Power Authority, White Plains, NY USA, and also with Manhattan College, Riverdale, NY 10471 USA (e-mail: Bruce. Fardanesh@nypa.gov).

Color versions of one or more of the figures in this paper are available online at http://ieeexplore.ieee.org.

Digital Object Identifier 10.1109/TPWRS.2010.2047031

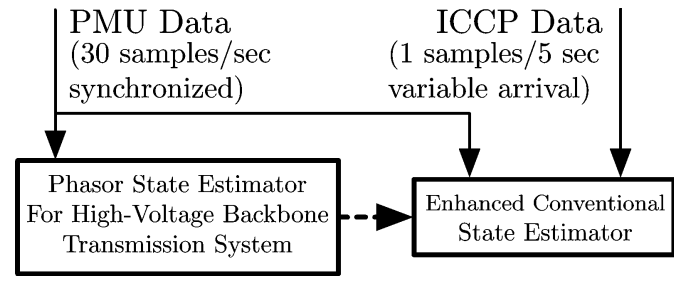

Fig. 1. Phasor state estimation supplementing conventional state estimation.

In the case when a PMU is applied to every bus on the system, [12] establishes a non-iterative least-squares solution to perform state estimation in rectangular coordinates. Assuming far fewer PMUs, [13] suggests to install PMUs at critical buses to enhance the reliability of conventional SEs. ${ }^{1}$

In this paper, we investigate a phasor state estimator (PSE) framework for power systems where a number of PMUs have been installed on high-voltage (HV) substations, although not necessarily on every HV substation. This new PSE will be built on synchronized phasor data only, and its solutions can be used to supplement a conventional SE based on ICCP data, as shown in Fig. 1. This approach is attractive because the PSE is built independently of the conventional SE, allowing the PSE to be implemented without disrupting the role of a conventional SE in control centers.

The proposed PSE uses polar coordinates in its problem formulation, which requires an iterative solution procedure. The polar coordinate formulation is a better choice than the rectangular coordinate formulation [12] because the magnitude and phase of a phasor quantity as measured and computed in a PMU are largely independent variables [14]. Consider the phasor diagram shown in Fig. 2, where $\tilde{V}_{1 m}=V_{1 m} \varepsilon^{j \theta_{1 m}}$ is the true voltage phasor, $\tilde{V}_{1 m}^{*}=V_{1 m} \varepsilon^{j\left(\theta_{1 m}+\Delta \theta\right)}$ is the measured phasor $\tilde{V}_{1}$ with an angle error of $\Delta \theta$, and $\tilde{e}=\tilde{V}_{1}-\tilde{V}_{1}^{*}$ is the corresponding error phasor. Instead of two error quantities $\Delta x$ and $\Delta y$ in rectangular coordinates, the angle error $\Delta \theta$ is a single variable in the polar coordinates.

These type of phase angle errors have been observed from recorded data in several utilities [15], [16], which may be due to a variety of reasons. Phasor angles are typically computed using some signal processing techniques over one cycle or portions of a cycle to minimize the impact of quantization. The phase is also affected by the length of potential and current transformer cables. External synchronization issues with respect to GPS receivers and internal synchronization issues due to computational burden may also induce time delays, which translate into a phase

${ }^{1}$ Loss of ICCP data at critical buses will result in unobservable islands. 


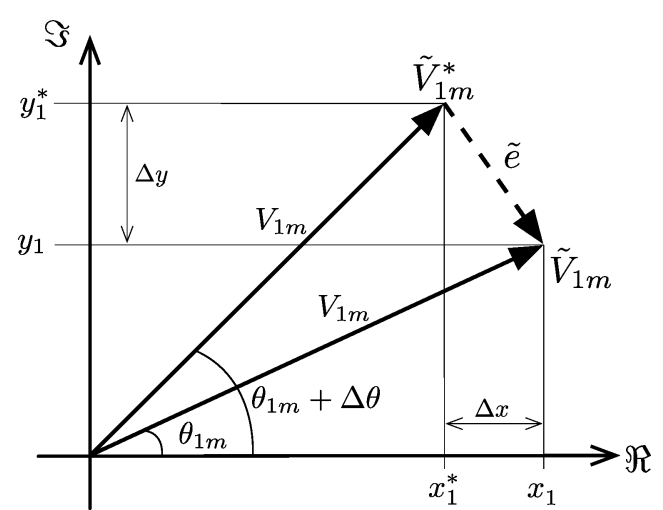

Fig. 2. Polar and rectangular coordinate representation of an angular error in a phasor measurement.

lag in the measured data. We have observed random phase jumps of multiples of $7.5^{\circ}$ and random saw-tooth behavior (as shown later in Section VI). Equally important, we have also observed that if such a phase bias occurs in a particular channel of a PMU, then the same bias will appear in all of the phase channels. ${ }^{3}$ Thus, the polar coordinates provide a preferred setting for using weighted least-squares (WLS) techniques to correct the biases in the measured phasor data. The phase bias is a form of bad data, which is not part of conventional SCADA data. Here the magnitude is assumed to be correct, although it is still subject to normal calibration accuracy.

Phasor angle and magnitude error correction requires a power network to have redundant PMU measurements. Loosely speaking, redundancy means that the voltage phasor of a non-PMU bus can be computed by PMU data from two different buses. Thus, compared to observability, redundancy requires more PMUs. A result on the number of PMUs for redundancy is provided in Section IV.

The remainder of this paper is organized as follows. In Section II, the formulation for the PSE model is presented. Section III introduces the phasor state estimation solution method and discusses observability conditions. In Section IV, the methodology is extended to provide phasor angle bias correction, and redundancy measurement conditions are derived. In Section V, an observability and redundancy analysis is carried out on several test networks. The application of the PSE to actual PMU data recorded in portions of the AEP's high voltage network is demonstrated in Section VI. Conclusions are presented in Section VII.

\section{Measurement Model in Polar CoORdinates}

We first provide the formulation of the PSE model. A bus or line that is measured by a PMU is called a PMU bus or a PMU line. Otherwise, it will be referred to as either a non-PMU bus or a non-PMU line.

${ }^{2} \mathrm{~A} 60-\mathrm{Hz}$ signal measured at 48 points per cycle will result in an error of $360^{\circ} / 48=7.5^{\circ}$ if a data point is skipped.

${ }^{3}$ All phase channels use the same GPS clock signal and identical digital signal processing code.

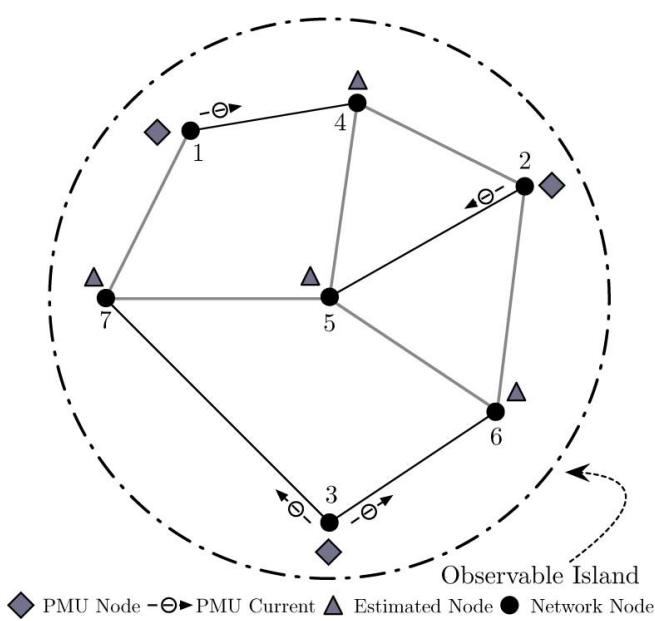

Fig. 3. PSE observable island (Network 1).

\section{A. Buses and Lines in the PSE Model}

Consider a power system with $N$ buses interconnected by $L$ lines. We label these buses as $i=1,2, \ldots, N$, and their corresponding bus voltage phasors as $\tilde{V}_{i}=V_{i} \varepsilon^{j \theta_{i}}, i=1,2, \ldots, N$. The phasor measurements for these voltages are denoted as $\tilde{V}_{i m}=V_{i m} \varepsilon^{j \theta_{i m}}, i=1,2, \ldots, N_{1}$.

The PMUs also measure a number $L_{1}$ of line currents between the buses. The current going from a PMU Bus $i$ to another Bus $k$, whether a PMU bus or a non-PMU bus, will be denoted by $\tilde{I}_{i k}=I_{i k} \varepsilon^{j \delta_{i k}}$, whose measurements will be denoted as $\tilde{I}_{i k m}=I_{i k m} \varepsilon^{j \delta_{i k m}}$. Let the total number of non-PMU buses be denoted by $N_{2}=N-N_{1}$, and without loss of generality, these buses will be labeled as $i=N_{1}+1, N_{1}+2, \ldots, N$. Similarly, let the number of non-PMU lines be $L_{2}=L-L_{1}$. Thus, the collection of these $N=N_{1}+N_{2}$ buses and $L=L_{1}+L_{2}$ branches connecting these buses comprises the PSE network model.

An example of such a PSE network is shown in Fig. 3. ${ }^{4}$ The voltage phasors are measured at the PMU Buses 1, 2, and 3. These PMUs also measure the current phasors on Lines 1-4, 2-5, 3-6, and 3-7. Thus, the non-PMU Buses 4, 5, 6, and 7, and Lines $1-7,2-4,2-6,4-5,5-6$, and 5-7 are included in the PSE network, which has a total of seven buses and ten lines. Note that connections from these seven buses to other buses outside this island are not needed.

\section{B. Measurement Model}

At each PMU bus, the available measurements are $V_{i m}, \theta_{i m}$, $I_{i k m}$, and $\delta_{i k m}$. The measurement equations are divided into voltage measurement equations and current measurement equations.

1) Voltage Measurement Equations: They are constructed by using the $V_{i m}$ and $\theta_{i m}$ measurements at each PMU bus and equating them to their corresponding states, resulting in

$$
V_{i}-V_{i m}=e_{V_{i}}, \quad \theta_{i}-\theta_{i m}=e_{\theta_{i}}
$$

where $e_{V_{i}}$ and $e_{\theta_{i}}$ are voltage measurement residuals.

\footnotetext{
${ }^{4} \mathrm{~A}$ formal definition for PSE Islands is given in Section III-D.
} 


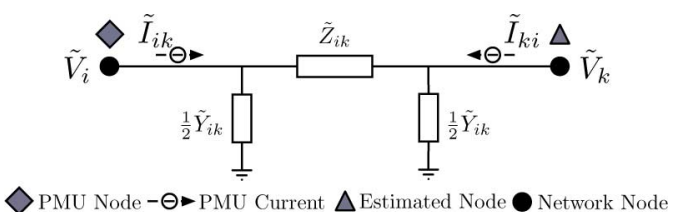

Fig. 4. PMU Bus- $i$ circuit for the PSE network model.

2) Current Measurement Equations: They are constructed by using the $I_{i k m}$ and $\delta_{i k m}$ measurements at each PMU bus for Line $i-k$ as

$$
I_{i k}-I_{i_{k} m}=e_{I_{i k}}, \quad \delta_{i k}-\delta_{i k m}=e_{\delta_{i k}}
$$

where $e_{I_{i k}}$ and $e_{\delta_{i k}}$ are current measurement residuals.

\section{Network Model}

The network model is developed based on the equivalent circuit in Fig. 4 connecting any two buses in the PSE network. The branch connecting Buses $i$ and $k$ is represented by the transmission line with impedance $\tilde{Z}_{i k}=R_{i k}+j X_{i k}$ and line charging $\tilde{Y}_{i k}=j B_{i k}$. The current phasor leaving Bus $i$ as $\tilde{I}_{i k}$ is related to the bus voltages and line parameters as

$$
\tilde{V}_{k}=\tilde{V}_{i}-\tilde{Z}_{i k}\left(\tilde{I}_{i k}-\frac{1}{2} \tilde{Y}_{i k} \tilde{V}_{i}\right) \cdot
$$

Note that there will be $L$ such complex equations for the PSE network. Each complex circuit equation can be decomposed into two equations, one for the real part and the other for the imaginary part, respectively, as

$$
f_{i k \mathrm{re}}=\operatorname{Re}\left(f_{i k}\right)=0, \quad f_{i k \mathrm{im}}=\operatorname{Im}\left(f_{i k}\right)=0
$$

where

$$
f_{i k}=\left(1+\frac{1}{2} \tilde{Y}_{i k} \tilde{Z}_{i k}\right) \tilde{V}_{i}-\tilde{Z}_{i k} \tilde{I}_{i k}-\tilde{V}_{k}
$$

We define the $2 L$ dimensional vector $f$

$$
f=\left[\begin{array}{llll}
\ldots & f_{i k \mathrm{re}} & f_{i k \mathrm{im}} & \ldots
\end{array}\right]^{T}
$$

of all the lines. Furthermore, the branches with current phasor measurements will occupy the first $2 L_{1}$ rows of $f$.

\section{State Vector Organization}

The total number of unknown variables in the PSE model are $2 N$ voltage magnitudes and angles, and $2 L$ line current magnitudes and angles. These unknown variables are arranged as a vector

$$
x=\left[\begin{array}{llll}
V^{T} & I^{T} & \theta^{T} & \delta^{T}
\end{array}\right]^{T}
$$

where the voltage magnitude and angle vectors are, respectively, defined as

$$
V=\left[\begin{array}{llllll}
V_{1} & \ldots & V_{N_{1}} & V_{N_{1}+1} & \ldots & V_{N}
\end{array}\right]^{T}
$$

$$
\theta=\left[\begin{array}{llllll}
\theta_{1} & \cdots & \theta_{N_{1}} & \theta_{N_{1}+1} & \ldots & \theta_{N}
\end{array}\right]^{T}
$$

and the current magnitude and angle vectors are, respectively, defined as

$$
\begin{aligned}
I & =\left[\begin{array}{lll}
\ldots & I_{i k} & \ldots
\end{array}\right]^{T} \\
\delta & =\left[\begin{array}{lll}
\ldots & \delta_{i k} & \ldots
\end{array}\right]^{T}
\end{aligned}
$$

for all of the lines where the measured current magnitudes and angles are placed in the first parts of these vectors. Note that we augment to the voltage state vector in a conventional SE, the line current variables, because it provides a framework to correct for current angle bias.

\section{Phasor State Estimation Solution AND OBSERVABILITY}

\section{A. Least-Squares Formulation}

The objective of the PSE problem is to find a set of voltage and current phasors satisfying (3) while minimizing the measurement errors in the measurement (1) and (2). The PSE can be formulated as a nonlinear weighted least-squares (WLS) problem [17], [18]. Define the objective function as

$$
q(x)=\frac{1}{2}\left(\left\|W_{V} e_{V}\right\|^{2}+\left\|W_{\theta} e_{\theta}\right\|^{2}+\left\|W_{I} e_{I}\right\|^{2}+\left\|W_{\delta} e_{\delta}\right\|^{2}\right)
$$

where $\|\cdot\|$ denotes the magnitude of its vector argument, $e_{V}, e_{\theta}$, $e_{I}$, and $e_{\delta}$ are vectors of the measurement residuals in (1) and (2). The weighting matrices in (12) are diagonal matrices given by

$$
\begin{aligned}
& W_{V}=\operatorname{diag}\left(\ldots, \min \left(1, \frac{1}{V_{i m}}\right), \ldots\right) \\
& W_{\theta}=\operatorname{diag}\left(\ldots, \min \left(1, \frac{1}{\left|\theta_{i m}\right|}\right), \ldots\right) \\
& W_{I}=\operatorname{diag}\left(\ldots, \min \left(1, \frac{1}{I_{i k m}}\right), \ldots\right) \\
& W_{\delta}=\operatorname{diag}\left(\ldots, \min \left(1, \frac{1}{\left|\delta_{i k m}\right|}\right), \ldots\right) .
\end{aligned}
$$

The weights are designed such that the normalized value for each variable is comparable to those of other variables so that measurement variance can be comparable.

The WLS problem can be formulated as

$$
\min _{x} q(x), \quad \text { subject to }: f=0 .
$$

Because $f$ is a nonlinear function of $V, I, \theta$, and $\delta$, we augment the equality constraint $f=0$ to the objective function $q(x)$ to form

$$
q^{\prime}(x)=q(x)+\frac{1}{2}\left\|W_{f} f\right\|^{2}=\frac{1}{2}\|W h(x)\|^{2}
$$

where $W_{f}$ is diagonal matrix with large weights on the network equations thus enforcing $f=0$

$$
h=\left[\begin{array}{ll}
f^{T} & e^{T}
\end{array}\right]^{T}, \quad e=\left[\begin{array}{llll}
e_{V}^{T} & e_{\theta}^{T} & e_{I}^{T} & e_{\delta}^{T}
\end{array}\right]^{T}
$$




$$
W=\operatorname{block}-\operatorname{diag}\left(\begin{array}{lllll}
W_{f} & W_{V} & W_{\theta} & W_{I} & W_{\delta}
\end{array}\right) .
$$

Thus, the constrained WLS problem (17) is transformed into an unconstrained WLS problem of

$$
\min _{x} q^{\prime}(x) .
$$

\section{B. Successive Solution Algorithms}

There are a number of approaches to solve the nonlinear WLS problem (21). A Newton algorithm would require the second derivative of $q^{\prime}$. Here we discuss the Gauss-Newton method, which requires only the first derivative of $q^{\prime}$.

In the Gauss-Newton method, starting from the current value $x_{c}$ of the solution vector $x$, the increment in $x$ is computed as

$$
\Delta x=-\left(H\left(x_{c}\right)\right)^{-1}\left(W J\left(x_{c}\right)\right)^{T} h\left(x_{c}\right)
$$

where the Jacobian matrix is

$$
J(x)=\frac{\partial h(x)}{\partial x}
$$

and

$$
H=\left(W J\left(x_{c}\right)\right)^{T}\left(W J\left(x_{c}\right)\right) .
$$

The new solution is updated to $x_{c}+\Delta x$ and the Gauss-Newton iteration (22) is repeated until the solution converges. Note that the convergence of the Gauss-Newton iteration is quadratic when the solution is close to the optimum value of $x$. Observe that by appropriately calculating the starting guess, $x_{0}$, a solution may be reached with only a few iterations.

\section{Jacobian Matrix Structure}

From the ordering of the unknown variables in $x$ (7) and the network (19), the structure of the Jacobian (23) has the following form:

$J=\left[\begin{array}{c|c|c|c|c|c|c|c}\hline U_{V} & 0 & 0 & 0 & 0 & 0 & 0 & 0 \\ \hline 0 & 0 & 0 & 0 & U_{I} & 0 & 0 & 0 \\ \hline 0 & 0 & U_{\theta} & 0 & 0 & 0 & 0 & 0 \\ \hline 0 & 0 & 0 & 0 & 0 & 0 & U_{\delta} & 0\end{array}\right]$

where the identity matrices $U_{V}$ and $U_{\theta}$ have a dimension of $N_{1} \times N_{1}$, and matrices $U_{I}$ and $U_{\delta}$ have a dimension of $L_{1} \times L_{1}$, arising from the partial derivatives of the measurement error vector with respect to its unknowns. The measurement equations are ordered as follows: voltage magnitudes, current magnitudes, voltage angles, and current angles. Therefore, the columns of $J$ are arranged corresponding to the state vector in (7), placing the states with measurements in the first part of each vector, as shown in (8)-(9). Algorithms [17] exploiting sparsity can be used to solve for the increment $\Delta x$ in (22) for computational efficiency.

\section{Observability Condition}

The phasor state estimation requires that $H$ (24) be nonsingular, that is, the Jacobian matrix has full rank and is equal to the number of unknown variables

$$
\operatorname{rank}(J)=2(N+L) .
$$

This rank condition is satisfied when the network is observable, similar to the notion used in conventional state estimation. Thus, starting from the PSE model, we can proceed to determine whether there are any observable islands within the network, using an observability algorithm [2], [3] to isolate the islands [19]. We define a PSE observable island as a portion of the power network for which condition (26) is satisfied. If full observability is required, the algorithms in [20]-[23] may be used for adding new PMUs. Throughout this paper, a PSE model is constructed for each island, such that all the voltages of the $N$ buses and the currents on the $L$ branches connecting them will be observable. The rank condition (26) can also be used to determine a lower bound on the number of phasor measurements needed. In the PSE model $U_{T}=2(N+L)$ unknown variables are constrained by $E_{T}=2\left(L+N_{1}+L_{1}\right)$ real equations arising from (3), (1), and (2). Thus for the PSE network to be observable, it is necessary that

$$
N_{1}+L_{1} \geq N
$$

The PSE network in Fig. 3 is observable with $N=7, N_{1}=3$, and $L_{1}=4$, satisfying (27).

In the case in which relay-based PMUs are used [22], [24], where one voltage phasor and one line current phasor are measured for each PMU, (27) reduces to $2 L_{1} \geq N$, that is

$$
L_{1} \geq \operatorname{int}\left[\frac{N}{2}\right]
$$

where $\operatorname{int}[N / 2]$ denotes an integer greater or equal to $N / 2$, provided that the PMUs are all on distinct buses. Equation (28) denotes the minimum number of current measurements required to make a PSE model observable.

More generally, when each PMU measures all incident currents [25] from a substation, the network becomes observable with fewer PMUs. It has been estimated in [20] that observability can be achieved with PMUs on $N / 4$ to $N / 3$ buses in the network.

\section{Extension for Phase Angle Shift CORRECTION AND REDUNDANCY}

\section{A. Example Systems With Redundancy}

As discussed in Section I, a particular characteristic observed from measured data is that when an angle shift error $\phi_{i}$ occurs in the $i$ th PMU, the same shift $\phi_{i}$ will appear in all measured voltage and current phasors in that PMU. To correct for this bias, we need the notion of redundancy.

To motivate, consider the two PSE networks shown in Fig. 5, each with the indicated voltage and current phasor measurements. In Fig. 5(a), $\tilde{V}_{3}$ can be computed using the phasor mea- 


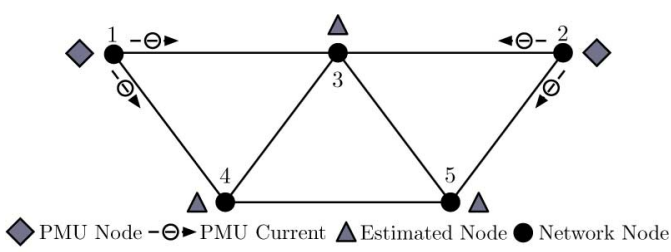

(a)

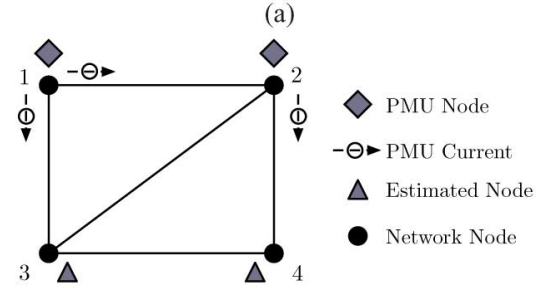

(b)

Fig. 5. Example networks allowing angle bias correction. (a) Five-bus PSE network (Network 2). (b) Four-bus PSE network (Network 3).

surements from either Bus 1 or Bus 2. If one of the phasor measurements has an angle bias, the two computed values of $\tilde{V}_{3}$ will be different. If the angle bias is corrected, then all five voltages and seven currents in the network can be accurately computed. A simpler example system in given in Fig. 5(b) where the voltage phasor measurements at adjacent buses provide a check on the phasor current. More detailed derivations will be available from [26].

\section{B. Modification of the Measurement Model}

Incorporating $\phi_{i}$ in the analysis, the measurement (1) and (2) can be updated to

$$
\theta_{i}=\theta_{i m}-\phi_{i}+e_{\theta_{i}}
$$

and

$$
\delta_{i k}=\delta_{i k m}-\phi_{i}+e_{\delta_{i k}}
$$

Note that we take Bus 1 to be the reference bus, and thus, the angle bias will not be applied to its phasor measurements. The angle bias terms form a vector

$$
\phi=\left[\begin{array}{llll}
\phi_{2} & \phi_{3} & \ldots & \phi_{N_{1}}
\end{array}\right]^{T} .
$$

Thus, the WLS algorithm (21) can be modified to the problem

$$
\min _{x_{\phi}} q^{\prime}\left(x_{\phi}\right)
$$

where

$$
x_{\phi}=\left[\begin{array}{ll}
x^{T} & \phi^{T}
\end{array}\right]^{T}
$$

and $e\left(x_{\phi}\right)$ has been modified to incorporate (29) and (30).

Note that the Jacobian matrix is expanded to

$$
J_{\phi}=\left[J \mid \frac{0}{\partial e(\phi) / \partial \phi}\right]
$$

where the Jacobian submatrix $\partial e(\phi) / \partial \phi$ is sparse and consists of ones and zeros.
TABLE I

OBSERVABILITY AND REDUNDANCY ANALYSIS RESULTS

OBSERVABILITY ANALYSIS

\begin{tabular}{|c|c|c|c|c|}
\hline NETWORK & $E_{T}$ & $U_{T}$ & $\operatorname{rank}(J)$ & OBSERVABLE \\
\hline 1 & 34 & 34 & 34 & YES \\
\hline 2 & 26 & 24 & 24 & YES \\
\hline 3 & 20 & 18 & 18 & YES \\
\hline
\end{tabular}

REDUNDANCY ANALYSIS

\begin{tabular}{|c|c|c|c|c|c|}
\hline NETWORK & BIAS TERMS & $E_{T}$ & $U_{T \phi}$ & $\operatorname{rank}\left(J_{\phi}\right)$ & REDUNDANT \\
\hline 1 & $\phi_{2}, \phi_{3}$ & 34 & 36 & 34 & NO \\
\hline 2 & $\phi_{2}$ & 26 & 25 & 25 & YES \\
\hline 3 & $\phi_{2}$ & 20 & 19 & 19 & YES \\
\hline
\end{tabular}

\section{Redundancy Condition}

To correct angle shifts, we require

$$
\operatorname{rank}\left(J_{\phi}\right)=U_{T \phi}
$$

where $U_{T \phi}=2(N+L)+\left(N_{1}-1\right)$ is the number of unknown variables which include the angle biases.

The rank condition (35) can also be used to determine a lower bound on the number of measurements needed for redundancy. A necessary condition is that the number of rows in (34), $E_{T}=$ $2\left(L+N_{1}+L_{1}\right)$, should be greater than or equal to the number $U_{T \phi}$ of unknowns, that is,

$$
N_{1}+2 L_{1} \geq 2 N-1 \text {. }
$$

To be more specific, the number of angle unknowns must be less than or equal to the number of angle equations in the $J_{\phi}$, i.e.,

$$
N+L+N_{1}-1 \leq L+N_{1}+L_{1}
$$

Simplifying the expression above yields in a lower bound on the number of current measurements

$$
L_{1} \geq N-1
$$

It is interesting to note that (37) is a condition on the number of line currents required, although implicitly each line current is accompanied by a measured voltage phasor. With phasor data on $N_{1}$ lines, one can connect all $N$ buses in the PSE model. With relay-based PMUs, redundancy requires $N-1$ PMUs with connectivity. For station-based PMUs, fewer PMUs would be needed, provided those PMUs have at least $N-1$ current measurements with connectivity.

\section{OBSERVABILITY AND REDUNDANCY ANALYSIS FOR TEST NETWORKS}

Next we perform a numerical observability and redundancy analysis on Network 1 (Fig. 3), Network 2 [Fig. 5(a)], and Network 3 [Fig. 5(b)], using the condition (26) for observability, and the condition (35) for redundancy. Table I summarizes the results for both analyses.

1) Observability: For all three networks, the rank of their Jacobian matrices $J$ is equal to the number of unknowns $U_{T}$, as shown in Table I, implying that they are all observable.

2) Redundancy: Now consider correcting potential biases on the PMU buses. For Network $1, \phi_{2}$ and $\phi_{3}$ are added, resulting in 


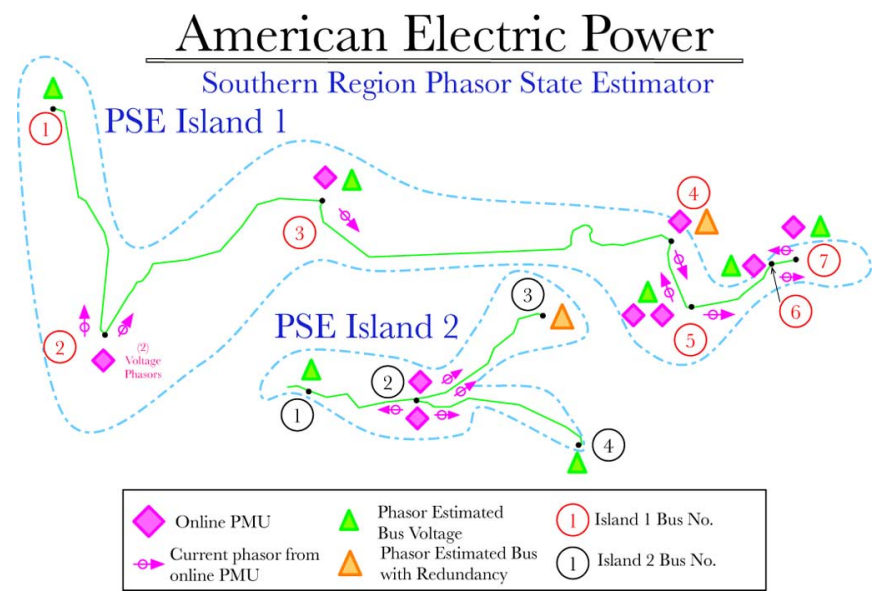

Fig. 6. AEP's 765-kV network and PSE islands.

a total of 36 unknowns. The number of network equations, however, remains at 34 , and the rank of $J_{\phi}$ is also 34 . Therefore, due to the lack of redundancy, PMU angle biases cannot be corrected for Network 1. For Networks 2 and 3, we include the angle bias term $\phi_{2}$. The number of unknowns is increased by one, and the rank of $J_{\phi}$ is also increased by one from $J$. Measurement redundancy in both networks allows reliable angle shift correction by satisfying condition (35).

\section{Illustration With AEP's High-Voltage Network}

In this section, we illustrate phasor state estimation with portions of American Electric Power's (AEP) southern HV network shown in Fig. 6, where we have highlighted two PSE Islands. The locations of the PMUs are marked in the figure. Using the bus voltage and current measurements shown in the diagram, we set up the measurement model and the Jacobian matrix for each island. The state estimation solution is implemented with a Gauss-Newton solution algorithm. These two islands are small, but serve to illustrate the main features of the PSE with real PMU data. We expect that these islands will grow when additional PMU coverage is available. The PSE model can be enhanced in stages as each new PMU is installed in AEP's system.

\section{A. PSE Island 1}

The network in Island 1 has $N=7$ buses and $L=6$ lines, and a combination of station-based and relay-based PMUs. The measurement model includes two voltage phasor measurements at Buses 2 and 5. At Bus 2, two current phasors are obtained from the same PMU, one for Line $2-1$ and the other for Line $2-3$. At Bus 5 , the voltage phasor is provided by two different PMUs, one also measuring the current for Line $5-4$ and the other the current for Line 5-6. All other measurements are indicated in Fig. 6. Accounting for the redundant measurements, we have $N_{1}=8$ and $L_{1}=8$. Thus, the Jacobian for the PSE model has $E_{T}=44$ rows. To estimate the angle biases, we add six angle shift unknowns, one at each PMU, with the exception of Bus 2 which is used as reference. Thus, there is a total of $U_{T \phi}=32$ unknowns. The augmented Jacobian, $J_{\phi}$, has $\operatorname{rank}\left(J_{\phi}\right)=32$
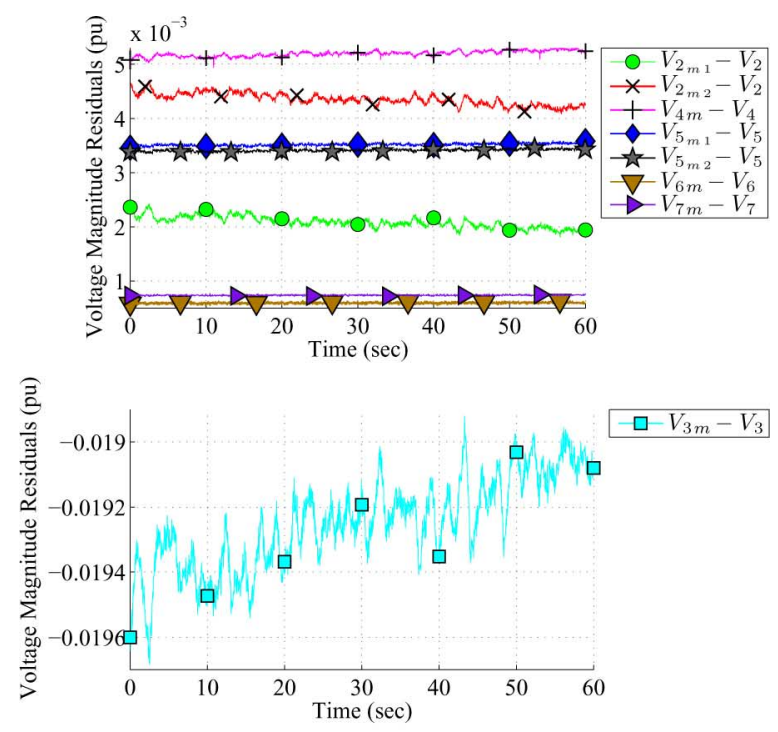

(a)
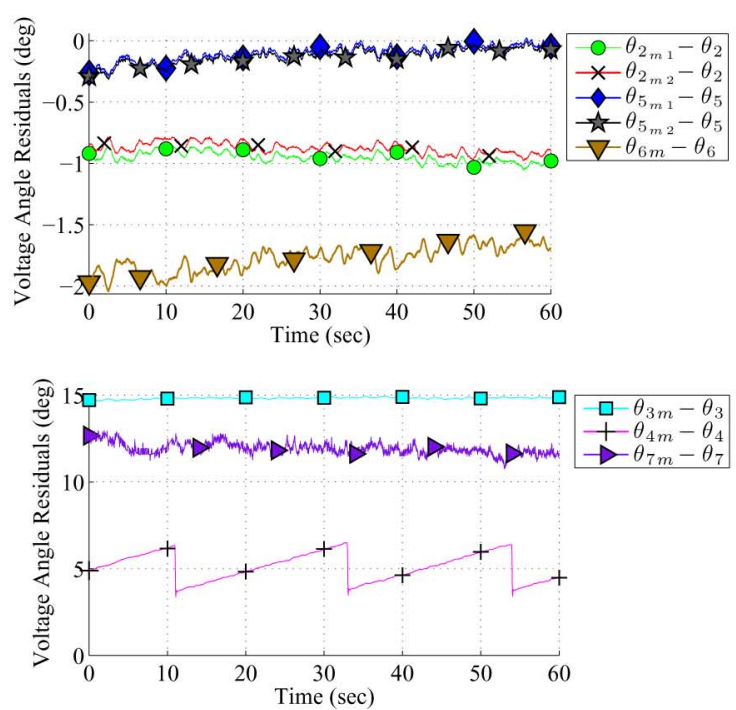

(b)

Fig. 7. Residuals for the bus voltage magnitudes and angles in Island 1. (a) Voltage magnitude residuals, $V_{i m}-V_{i}$. (b) Voltage angle residuals, $\theta_{i m}-\theta_{i}$.

satisfying (35), and therefore allowing for angle-bias correction within Island 1.

\section{B. PSE Island 2}

The network in Island 2 has $N=4$ buses and $L=3$ lines, and one station-based and one relay-based PMU. The voltage phasor at Bus 2 is measured by both PMUs. The current measurements made by the PMUs are indicated in Fig. 6. Accounting for the redundant measurements, we have $N_{1}=2$ and $L_{1}=4$. Thus, the Jacobian for the PSE model has $E_{T}=18$ rows. To estimate angle bias, one angle shift unknown is assigned to the relay-based PMU measuring Line $2-3$, thus allowing correction of phase errors in measurements $\theta_{2 m_{2}}$ and $\delta_{23 m_{2}}$. Hence, there is a total of $U_{T \phi}=15$. The augmented Jacobian in Island 2 has $\operatorname{rank}\left(J_{\phi}\right)=15$, satisfying condition (35) and allowing angle bias correction. 


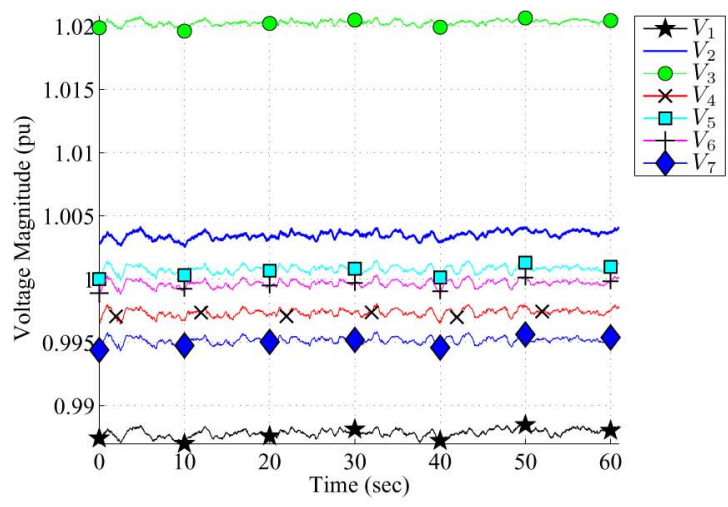

(a)

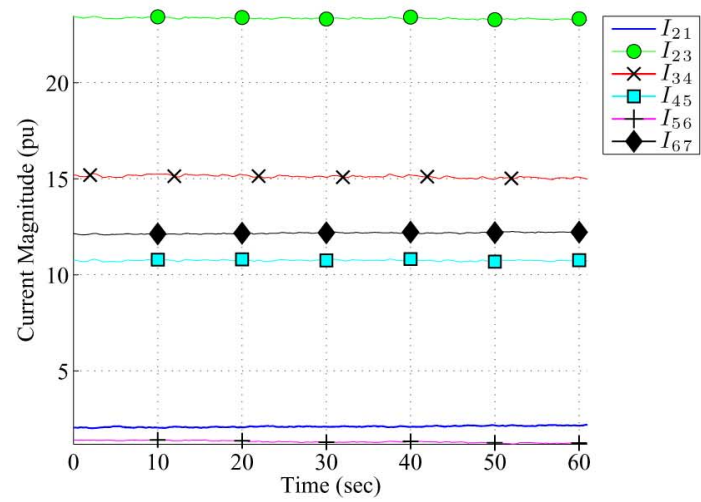

(b)

Fig. 8. Island 1 voltage and current magnitude estimates. (a) Island 1 estimated voltage magnitudes. (b) Island 1 estimated current magnitudes.

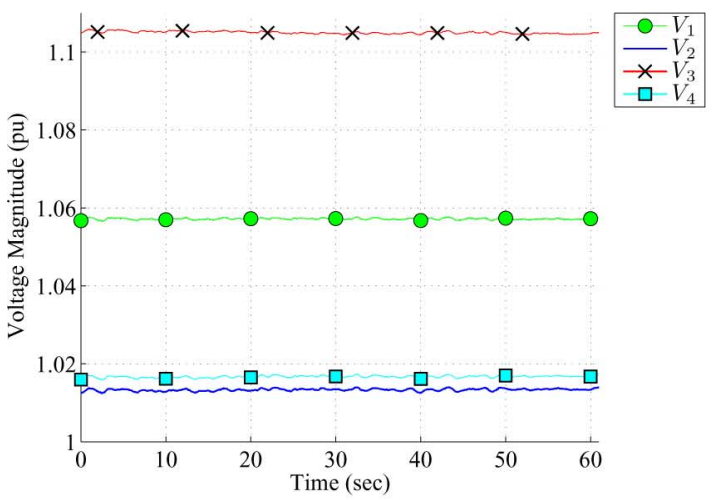

(a)

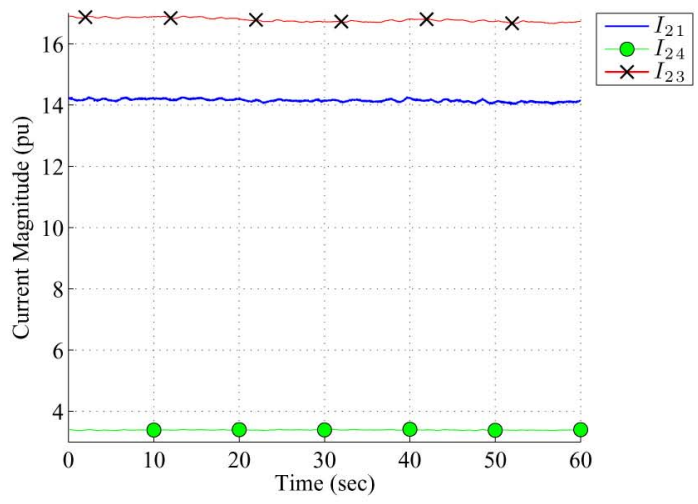

(b)

Fig. 9. Island 2 voltage and current magnitude estimates. (a) Island 2 estimated voltage magnitudes. (b) Island 1 estimated current magnitudes.
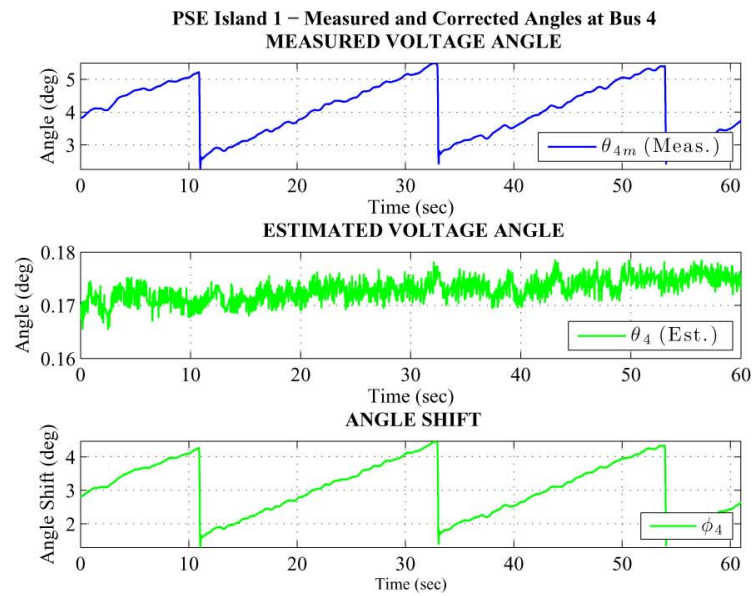

(a)
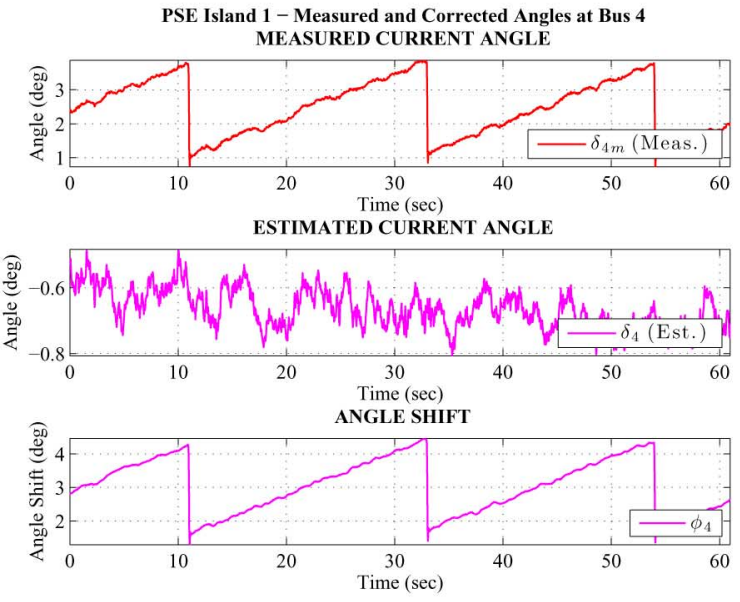

(b)

Fig. 10. Voltage and current angle measurements, estimates, and angle shift in island 1. (a) Voltage angle measurement, estimate, and shift in island 1. (b) Current angle measurements, estimate, and shift in island 1.

\section{State Estimation and Phase Angle Correction}

The PSE models for Islands 1 and 2 are implemented in a Gauss-Newton algorithm. The algorithm uses all the available measurement data and solves sequentially for each set of measurements. We have used a data set that illustrates the capability for angle correction. The resulting estimates provided by the PSE are shown in Figs. 8-11, and the measurement residuals for the voltage magnitudes, $e_{V_{i}}=V_{i m}-V_{i}$, and voltage angles, $e_{\theta_{i}}=\theta_{i m}-\theta_{i}$, are shown in Fig. 7(a) and (b), respectively.

Note from Fig. 7(a) that the voltage magnitude residuals are consistent with what we would normally expect as a result from instrument calibration errors. Angle biases are significant in some channels, possibly due to firmware and software issues in obtaining the phase of a measured quantity as shown in the voltage angle residuals in Fig. 7(b).

Observe from Fig. 10 that the voltage and current phasors measured at Bus 4 present an angle error. The voltage angle measurement shows an undesirable saw-tooth behavior-a slew with a periodic reset. Likewise, in Island 2, the Bus 2 PMU measurements also exhibit a similar behavior, as shown in Fig. 11. The voltage angle measurement has an average value of about 

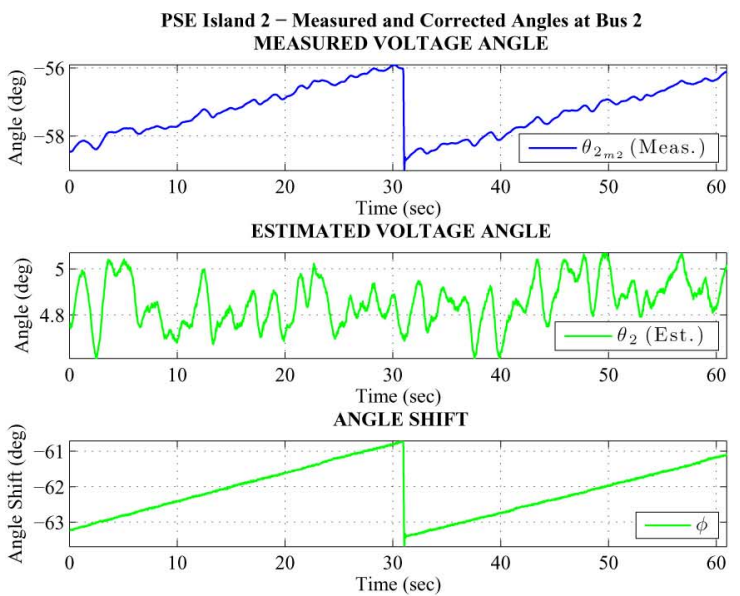

(a)
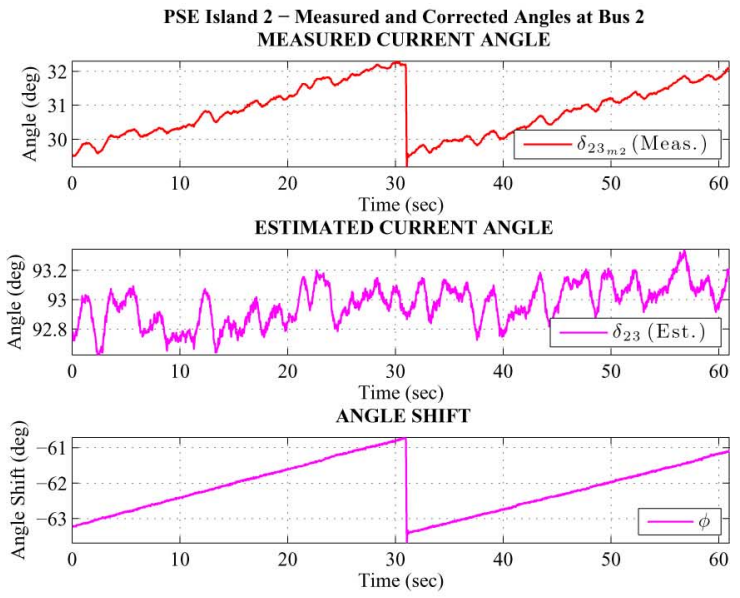

(b)

Fig. 11. Voltage and current angle measurements, estimates, and angle shift in island 2. (a) Voltage angle measurement, estimate, and shift in island 2. (b) Current angles measurements, estimates, and shift in island 2.

$-57^{\circ}$. Regardless of these measured phase angle errors, the PSE algorithm is capable of correcting for them. Figs. 10 and 11 show the estimated angle and the angle-bias term which accounts for the necessary correction at each bus, while Figs. 8 and 9 show the estimates for all voltage magnitude and current magnitude state variables. We should stress that without the angle bias correction capabilities of the PSE method, the estimation process will converge to invalid solutions.

The method has also been applied to a transfer path in New York's high-voltage system [16] using real PMU data where we deliberately introduced artificial angle shifts to test our methodology. When angle shifts were introduced, the increase in the number of iterations in the Gauss-Newton method was insignificant.

\section{CONCLUSIONS}

In this paper, we have developed a new least-squares approach to state estimation in the polar coordinate setting using synchronized voltage and current phasor data. The formulation readily extends to the automatic detection and correction of angle biases that may exist in the measured data. The notion of PMU data redundancy to remove angle biases is introduced, and a redundancy condition in terms of the rank of a Jacobian matrix is developed. A discussion on the minimum number of line current phasors required for redundancy is provided. The PSE approach is demonstrated using measured phasor data from a portion of the AEP HV network, and is shown to be able to correct angle shifts in the measured data.

We would like to point out that the PSE can be deployed in stages as more PMUs are installed in power systems. Initially PSE can be based on observability. When redundancy becomes available, angle bias estimate can be added to the redundant part. Results from the PSE will improve the data quality for subsequent applications such as the conventional state estimator.

\section{REFERENCES}

[1] F. Schweppe and E. Handschin, "Static state estimation in electric power systems," Proc. IEEE, vol. 62, no. 7, pp. 972-982, 1974.

[2] A. Monticelli, State Estimation in Electric Power Systems: A Generalized Approach. New York: Springer, 1999.

[3] A. Abur and A. G. Expósito, Power System State Estimation: Theory and Implementation. Boca Raton, FL: CRC, 2004.

[4] J. Allemong, L. Radu, and A. Sasson, "A fast and reliable state estimation algorithm for AEP's new control center," IEEE Trans. Power App. Syst., vol. PAS-101, no. 4, pp. 933-944, Apr. 1982.

[5] H. Merrill and F. Schweppe, "Bad data suppression in power system static estimation," IEEE Trans. Power App. Syst., vol. PAS-90, no. 6, pp. 2718-2725, Nov. 1971.

[6] A. Phadke and J. Thorp, Synchronized Phasor Measurements and Their Applications. New York: Springer, 2008.

[7] I. W. Slutsker et al., "Implementation of phasor measurements in state estimator at Sevillana de Electricidad," in Proc. IEEE Power Industry Computer Application Conf., May 1995, pp. 392-398.

[8] B. Fardanesh, Use of Phasor Measurements in a Commercial (or Industrial) State Estimator, EPRI, Palo Alto, CA, 2004, Final Rep. 1011002.

[9] M. Parashar et al., Implementation of Phasor Measurements in SDG\&E State Estimator, California Energy Commission, Tech. Rep., 2008, PIER Program Energy Commission-1500-02-04 MR053.

[10] R. Avila-Rosales, M. J. Rice, J. Giri, L. Beard, and F. Galvan, "Recent experience with a hybrid SCADA/PMU on-line state estimator," in Proc. IEEE Power and Energy Soc. General Meeting, Jul. 2009.

[11] A. Ghassemian and B. Fardanesh, "Phasor assisted state estimation for NYS transmission system-Implementation and testing," in Proc. IEEE/PES Power Systems Conf. Expo., Mar. 2009, pp. 1-8.

[12] A. G. Phadke, J. S. Thorp, and K. J. Karimi, "State estimation with phasor measurements," IEEE Trans. Power Syst., vol. 1, no. 1, pp. 233-238, Feb. 1986.

[13] J. Chen and A. Abur, "Placement of PMUs to enable bad data detection in state estimation," IEEE Trans. Power Syst., vol. 21, no. 4, pp. 1608-1615, Nov. 2006.

[14] K. Martin, J. Hauer, and T. Faris, "PMU testing and installation considerations at the Bonneville power administration," in Proc. IEEE Power Eng. Soc. General Meeting, Jun. 2007, pp. 1-6.

[15] L. Vanfretti, J. H. Chow, S. Sarawgi, and D. Ellis, "Phasor state estimation," in Proc. North Amer. SynchroPhasor Initiative (NASPI) Work Group Meeting, Charlotte, NC, Oct. 16-17, 2008. [Online]. Available: http://www.naspi.org/meetings/workgroup/workgroup.stm.

[16] L. Vanfretti, J. H. Chow, S. Sarawgi, D. Ellis, and B. Fardanesh, "A framework for estimation of power systems based on synchronized phasor measurement data," in Proc. IEEE/PES General Meeting, Jul. 2009, pp. 1-6.

[17] A. Bjorck, Numerical Methods for Least Squares Problems. Philadelphia, PA: SIAM, 1996.

[18] S. Nash and A. Sofer, Linear and Nonlinear Programming. New York: McGraw-Hill, 1996.

[19] A. Monticelli and F. Wu, "Network observability: Identification of observable islands and measurement placement," IEEE Trans. Power App. Syst., vol. PAS-104, no. 5, pp. 1035-1041, May 1985.

[20] T. Baldwin, L. Mili, M. B. Boisen Jr., and R. Adapa, "Power system observability with minimal phasor measurement placement," IEEE Trans. Power Syst., vol. 8, no. 2, pp. 707-715, May 1993. 
[21] B. Xu and A. Abur, "Observability analysis and measurement placement for systems with PMUs," in Proc. IEEE PES Power Systems Conf. Expo., Oct. 2004, vol. 2, pp. 943-946.

[22] R. Emami and A. Abur, "Reliable placement of synchronized phasor measurements on network branches," in Proc. IEEE/PES Power Systems Conf. Expo., Mar. 2009, pp. 1-6.

[23] G. Krumpholz, K. Clements, and P. Davis, "Power system observability: A practical algorithm using network topology," IEEE Trans. Power App. Syst., vol. PAS-99, no. 4, pp. 1534-1542, Jul. 1980.

[24] R. Emami, A. Abur, and F. Galvan, "Optimal placement of phasor measurements for enhanced state estimation: A case study," in Proc. 16th Power Systems Computation Conf. (PSCC), Jul. 2008, pp. 1-6.

[25] Macrodyne Model 1690 PMU Disturbance Recorder. Clifton Park, NY, Macrodyne.

[26] L. Vanfretti, "Phasor measurement-based state estimation of electric power systems and linearized analysis of power system network oscillations," Ph.D. dissertation, Rensselaer Polytechnic Inst., Troy, NY, 2009.

Luigi Vanfretti (S'03-M'10) received the M.S. and Ph.D. degrees, both in electric power engineering, from Rensselaer Polytechnic Institute, Troy, NY, in 2007 and 2009, respectively.

He is a Post-Doctoral Research Associate at the Electrical, Computer, and Systems Engineering Department at Rensselaer Polytechnic Institute. He was a visiting researcher at the Department of Electronics and Electrical Engineering of The University of Glasgow, Glasgow, U.K., during Fall 2005. His research interests are modeling, dynamics, stability, and control of power systems; applications of PMU data; and open source software for power system engineering.
Joe H. Chow (F'92) received the M.S. and Ph.D. degrees from the University of Illinois, Urbana-Champaign.

After working in the General Electric Power System business in Schenectady, NY, he joined Rensselaer Polytechnic Institute, Troy, NY, in 1987. He is a Professor in the Electrical, Computer, and Systems Engineering Department and the Associate Dean of Engineering for Research and Graduate Programs. His research interests include multivariable control, power system dynamics and control, voltage-sourced converter-based FACTS controllers, and synchronized phasor data.

Sanjoy Sarawgi (M'03) received the B.Tech. (Hons.) degree from the Indian Institute of Technology, Kharagpur, India, in 2002 and the M.S. degree from Washington State University, Pullman, in 2004.

Since 2004, he has been with the Advanced Transmission Studies and Technologies section of American Electric Power, Columbus, $\mathrm{OH}$.

Mr. Sarawgi is a member of the IEEE Power \& Energy Society (PES).

Behruz (Bruce) Fardanesh (SM'07) received the B.S. degree in electrical engineering from Sharif University of Technology, Tehran, Iran, in 1979 and the M.S. and D.Eng. degrees, both in electrical engineering, from the University of Missouri-Rolla and Cleveland State University, Cleveland, OH, in 1981 and 1985 , respectively.

Since 1985 he has been teaching at Manhattan College, Riverdale, NY, where he holds the rank of Associate Professor of electrical engineering. Currently, he is also working in the area of Advanced Power Delivery in Research and Technology Development at the New York Power Authority, White Plains. His areas of interest are power systems dynamics, control, and operation. 